\title{
Middle Atlantic Census Division
}

National Cancer Institute

\section{Source}

National Cancer Institute. Middle Atlantic Census Division. NCI Thesaurus. Code C76339.

A census division of the United States consisting of New Jersey, New York, and

Pennsylvania. 